\title{
Saúde planetária: conclamação para a ação dos médicos de família de todo o mundo
}

Planetary health: a call to action of family doctors from around the world

\section{Salud planetaria: Llamada a la acción de los médicos de familia de todo el mundo}

Mayara Floss ${ }^{1} \oplus$, Enrique Falceto Barros ${ }^{2}(0$

${ }^{1}$ Grupo Hospitalar Conceição; Rural Seeds. Porto Alegre, RS, Brasil.

${ }^{2}$ Clínica da Família Teewald (ESF/SUS); Working Party on the Environment da World Organization of Family Doctors (WONCA).

Santa Maria do Herval, RS, Brasil.

\section{Resumo}

A WONCA, em conjunto com a Planetary Health Alliance, lançou uma conclamação para a ação dos MFCs do mundo pela saúde planetária. Este comentário discute os efeitos das mudanças ambientais e a conclamação em relação o papel do médico de família na perspectiva da saúde planetária.

Palavras-chave: Saúde Ambiental; Medicina de Família e Comunidade; Mudança Climática

\section{Abstract}

WONCA, with the Planetary Health Alliance, issued a declaration calling family doctors of the world to act on planetary health. This commentary discusses environmental changes and the call in relation to the role of the family doctor in the perspective of planetary health.

Keywords: Environmental Health; Family Practice; Climate Change

\section{Resumen}

WONCA, junto con la Planetary Health Alliance, lanzó un llamamiento a la acción de los medicos de familia del mundo por la salud planetaria. Este comentario discute los efectos de los cambios ambientales y el llamamiento y el papel del médico de familia en la perspectiva de la salud planetaria.

Palabras clave: Salud Ambiental; Medicina Familiar y Comunitaria; Cambio Climático

Autor correspondente: Mayara Floss.

E-mail: mayarafloss@ @otmail.com Fonte de financiamento: declaram não haver.

Parecer CEP: não se aplica.

Procedência e revisão por pares: revisado por pares.

Recebido em: 22/02/2019. Aprovado em: 26/02/2019.
Como citar: Floss M, Barros EF. Saúde planetária: conclamação para a ação dos médicos de família de todo o mundo. Rev Bras Med Fam Comunidade. 2019;14(41):1992. https://doi.org/10.5712/rbmfc14(41)1992

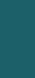

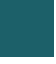


When the next generation asks us, "What did you do about climate change?," we want to have a good answer. ${ }^{1}$

Em 2017 a Organização Mundial dos Médicos de Família (WONCA) publicou a Declaração do WONCA sobre Saúde Planetária e Objetivos de Desenvolvimento Sustentável, ${ }^{2}$ importante marco institucional elevando Saúde Planetária há uma área prioritária para os médicos de família e comunidade (MFC). Desde então, as evidências científicas dos impactos da poluição ambiental sobre a saúde humana consolidaram-se.

Sabemos hoje que doenças causadas pela poluição foram responsáveis por cerca de 9 milhões de mortes prematuras em 2015, mais que três vezes as mortes de AIDS, malária e tuberculose. ${ }^{3}$ Ampliando ainda mais nosso olhar, observamos que os sistemas naturais (clima, terra, oceanos, biodiversidade, etc) que dão suporte para a saúde humana estão rapidamente se deteriorando, tornando-se evidente de que é preciso ação urgente para evitar que esse eminente colapso cause reversão das tendências de melhora global da saúde das últimas décadas. Por isso, a WONCA, em conjunto com a Planetary Health Alliance, lançou uma conclamação para a ação dos MFCs do mundo pela saúde planetária. ${ }^{4}$ Como generalistas ligados ao cotidiano das pessoas, e acostumados a liderar questões de equidade em saúde e a separar o joio do trigo, não espanta que estejamos liderando e soando o alarme global, pelo imperativo por ações em saúde planetária e mudança climática. ${ }^{5}$

A resposta inefetiva e insuficiente às mudanças climáticas colocam a vida humana em risco. ,6-8 $^{4,6}$ Eventos relacionados ao clima, como enchentes e secas, foram associados a mais de $90 \%$ de todos os desastres em todo o mundo nos últimos 20 anos. ${ }^{6}$ Bem como, no contexto brasileiro, doenças infecciosas como dengue também tem sua capacidade vetorial potencializada por mudanças climáticas, entre 1950 e 2010 a capacidade vetorial do Aedes aegypti teve um aumento de 5,8\% e para o Aedes albopictus $11,2 \% .{ }^{8} \mathrm{~A}$ poluição do ar é uma das principais causas de morte e incapacidade em todo o mundo, e estima-se que em 2015 cerca de 52 mil pessoas morreram no Brasil devido à exposição à poluição do ar ambiente..$^{3,8}$ Sendo que no mundo $90 \%$ das cidades respiram ar poluído e tóxico para o sistema cardiovascular e respiratório. ${ }^{7}$ O estresse por calor também é outra grande questão dentro do nosso país, entre 2014 e 2015 as ondas de calor duraram mais tempo no Brasil e isso pode ser mais danoso principalmente para para os idosos, crianças, gestantes e pessoas com doenças crônicas. ${ }^{6,7}$

A saúde planetária pode ser definida como um campo que investiga as interdependências entre a saúde dos sistemas naturais do planeta e a saúde da civilização humana. Destina-se a desenvolver e avaliar soluções baseadas em evidências para salvaguardar um mundo equitativo, sustentável e saudável. ${ }^{2}$ Nesse sentido a pergunta da declaração:4 "Por que os MFCs devem se preocupar sobre a Saúde Planetária?" coloca o papel do MFC agindo próximo das suas comunidades como um ator essencial tanto na mitigação quanto na adaptação aos problemas ambientais, buscando potencializar a saúde de pacientes com medidas que promovem co-benefícios ao ambiente como incentivar transporte ativo e alimentação saudável principalmente com foco em dieta baseada em plantas, ${ }^{9,10}$ entre outros. Mcwhinney, ${ }^{11}$ importante importante pensador de nossa disciplina médica, já falava do papel crucial do médico na defesa do ambiente saudável para seus pacientes. Finalmente, se refletirmos sobre os clássicos atributos da APS, de Barbara Starfield, ${ }^{12}$ perceberemos que os atributos derivados nos induzem a ampliar nosso olhar progressivamente para a família, para a comunidade, para a cultura, e por que não para os sistemas naturais que nos dão sustentação? 
Enfim, desafios globais necessitam de respostas globais; por conseguinte o WONCA - representando cerca de 500 mil médicos de família mundialmente - é uma força muito significativa no enfrentamento e na liderança pela saúde de nossas comunidades e de nosso planeta. Mas essa força formidável, só será efetiva se nos familiarizarmos e nos comprometermos com a Saúde Planetária no nosso cotidiano.

\section{Conflito de interesses}

Declaram não haver.

\section{Contribuição dos autores}

Concepção e/ou delineamento do estudo: MF, ECF. Aquisição, análise ou interpretação dos dados: MF, ECF. Redação preliminar: MF, ECF. Revisão crítica da versão preliminar: MF, ECF.

Todos os autores aprovaram a versão final e concordaram com prestar contas sobre todos os aspectos do trabalho.

\section{Referências}

1. Solomon CG, LaRocque RC. Climate Change - A Health Emergency. N Engl J Med. 2019;380:209-11. https://doi.org/10.1056/ NEJMp1817067

2. WONCA. Declaração do WONCA sobre Saúde Planetária e Objetivos de Desenvolvimento Sustentável; 2017. Disponível em: https://www.wonca.net/site/DefaultSite/filesystem/documents/Groups/Environment/planetary\%20health\%20espanol.pdf

3. Landrigan PJ, Fuller R, Acosta NJR, Adeyi O, Arnold R, Basu NN, et al. The Lancet Commission on pollution and health. Lancet. 2018 Feb 3;391(10119):462-512. https://doi.org/10.1016/S0140-6736(17)32345-0

4. WONCA. Declaration calling Family Doctors of the world to act on Planetary Health; 2019. Disponível em: http://www.globalfamilydoctor. com/Decplanethealth

5. Haines A, Ebi K. The Imperative for Climate Action to Protect Health. N Engl J Med. 2019(380):263-73. https://doi.org/10.1056/ NEJMra1807873

6. Watts N, Adger W, Ayeb-Karlsson S, Bai Y, Byass P, Campbell-Lendrum D. The Lancet Countdown: tracking progress on health and climate change. Lancet. 2017;389:1151-64. https://doi.org/10.1016/S0140-6736(16)32124-9

7. Watts N, Ammann M, Arnell N, Ayeb-Karlsson S, Belesova K, al. e. The 2018 Report of The Lancet Countdown on Health and Climate Change. The Lancet. 2018. https://doi.org/10.1016/S0140-6736(18)32594-7

8. Floss M, Barros E, Bressel M, Hacon S, Stein A, Sirena S, et al. Lancet Countdown 2018 Report: Briefing for Brazilian Policymakers Lancet Countdown. 2018;1:19. Disponível em: http://www.lancetcountdown.org/media/1417/2018-lancet-countdown-policy-brief-brazil. pdf

9. EAT-Lancet Commission Brief for Healthcare Professionals - EAT [Internet]. EAT. [cited 2019 Feb 13]. Disponível em: https://eatforum. org/lancet-commission/healthcare-professionals/

10. Xie E, Barros EFD, Abelsohn A, Stein AT, Haines A. Challenges and opportunities in planetary health for primary care providers. The Lancet Planetary Health 2018; 2. https://doi.org/10.1016/S2542-5196(18)30055-X

11. McWhinney IR. Family Medicine in Perspective. New England Journal of Medicine. 1975;293:176-81. Disponível em: https://www. aafpfoundation.org/content/dam/foundation/documents/who-we-are/cfhm/classicsfamilymedicine/FMPerspectiveMcWhinney.pdf

12. Starfield B. Atenção primária: equilíbrio entre necessidades de saúde, serviços e tecnologia. Brasília: UNESCO, Ministério da Saúde; 2002. 